\title{
BDNF integrity in ageing and stress
}

\section{Editorial}

Alzheimer's disease (AD) presents a progressive, stage-dependent age-related neurodegenerative disorder that is characterized by aggregation of toxic forms of amyloid $\beta$ peptide $(A \beta)$. There is much support for the notion that that brain-derived neurotrophic factor (BDNF) mediates beneficial effects of exercise on neuroplasticity and cellular stress resistance. ${ }^{1-3}$ BDNF is associated with neuroplasticity changes promoting health and well-being whereas these influences may be opposed by pro-inflammatory cytokines, key factors in neurodegenerative processes.$^{4}$ Lower serum levels are linked to greater symptom, cognitive, impairment. ${ }^{5}$ Thus, interventions, as for example physical exercise, whether acute or chronic, endurance or resistance, promote the mobilization of BDNF and other neurotrophic factors ${ }^{6,7}$ increase hippocampal and other brain regional resilience ${ }^{8}$ and therewith protect against cell death by inducing cell proliferation and maturation with enhanced neuronal reparation, neurogenesis and the growth and functioning of neurons in neurodegenerative disorders. ${ }^{9,10}$ Sampaio et al. ${ }^{11}$ in a review of the therapeutic applications of neurotrophic factors, particularly BDNF, postulate that they are essential for survival, development and maintenance of neurons of necessity for hindering the progress of impeding neurodegenerative staging in Alzheimer's disease (AD) and other diseases of the ageing process. Disturbances/ adversity in relation to the BDNF gene e.g. Val66Met single nucleotide polymorphism, exacerbates $\mathrm{AD}$ symptoms and biomarkers and other neurodegenerative brain disorders. ${ }^{12}$ Taking these aspects into consideration, with a middle-aged cohort presenting enhanced risk-for- $\mathrm{AD}$, the evidence showed that the carriage of the BDNF Met allele was associated with a sharper decline of episodic memory performance and executive function expressions and this decline was aggravated by the greater weight of the beta-amyloid biomarker (ibid). Furthermore, the reduced levels of BDNF in AD have been shown patients in comparisons with healthy control volunteers. ${ }^{13}$ In this respect, gender was shown to moderate the relationship between $\mathrm{A} \beta$, APOE genotype, and BDNF genotypes in predicting the severity of anxiety and depressive symptoms in cognitively normal older adults. ${ }^{14}$ Invariably, therapies increasing BDNF enhance resistance to $\mathrm{AD}$ : thus, keto-therapeutics promote mitochondrial respiration, advance neuronal long-term potentiation, increase BDNF expression, elevate GPR signaling, reduce oxidative stress, reduce inflammation, and induce protein post-translational modifications via lysine acetylation and $\beta$-hydroxybutyrylation ${ }^{15}$ while glial mGlu3R activation against $A \beta$ neurotoxicity through stimulating the release of protective neurotrophins, e.g. secreted amyloid precursor protein- $\alpha$ and BDNF, and promotes amyloid removal from extracellular space by glia-mediated phagocytosis. ${ }^{16}$ Loss of BDNF integrity is linked to oxidative stress and white matter regional impediments with late onset bipolar disorder and AD. ${ }^{17}$ Physical exercise induces several positive alterations in the human epigenome, which have the potential to enhance cognitive and psychological health, improve muscular fitness, and lead to better ageing with improved quality of life in older age.$^{18}$ In this regard, the physical exercise intervention has been shown repeatedly to alleviate symptom profiles and advance BDNF properties. $^{19}$

The integrity, under a variety of intrinsic and extrinsic conditions, of hippocampal functioning and cellular intactness, during old age
Volume I Issue 6 - 2017

Trevor Archer
Department of Psychology, University of Gothenburg, Sweden

Correspondence: Trevor Archer, Department of Psychology, University of Gothenburg, Sweden,

Email trevor.archer@psy.gu.se

Received: July 16,2017 | Published: August 01, 2017

progression under conditions of stress when confronted critical life episodes or periods, appears to be modulated by the availability and accessibility of BDNF. ${ }^{20-22}$ Over a wide range of neurologic and psychiatric disease states it is found that the integrity and concentrations of brain, serum and plasma BDNF is compromised, particularly under stressful conditions. Patients presenting mild cognitive impairment and dementia evidence lower levels of BDNF in serum than that expressed by healthy control individuals; concurrently, patients with dementia expressed smaller hippocampal volumes than the mild cognitive impairment patients and healthy control individuals. ${ }^{23}$ It has been found that acute forced-swim exposure or high-light openfield exposure induced a stressor effect that caused an elevation in the density of BDNF-immunoreactive pyramidal neurons, possibly relating to the up-regulation of hypothalamus-pituitary-adrenal axis mobilization and short time memory processing associated with the stressful situation. ${ }^{24}$ Under conditions of provoked toxicity, BDNF is reduced yet several interventions may reverse these effects: e.g. administration of methanol, which induces retinal toxicity, produced a reduction in the number of retinal ganglion cell s, loss of neurons, neuronal nuclear antigen, activation of glial fibrillary acidic proteinexpressing cells, suppression of BDNF+ positive cells, increase in apoptosis and enhancement of nitric oxide release in serum and brain. ${ }^{25}$ However, all these expressions of toxicity were reversed by light-emitting diode (LED) therapy which reduced markedly retinal ganglion cell death, compared with methanol-treated rats and increased notably the number of BDNF positive cells in the visual cortex of LED-treated group, compared with the methanol-intoxicated and control groups. In a study of patients presenting moderate $\mathrm{AD}$, the effects of a comprehensive intervention on plasma BDNF (pBDNF) and ADAS-Cognitive scores were assessed immediately after and six and 24 months later. ${ }^{26}$ At the time just after the comprehensive intervention both pBDNF and ADAS-Cognitive scores were reduced significantly, but while the latter worsened, the former increased versus baseline implying that may be a stressful event that prevented a return to homeostasis. Lead poisoning, mediated by enhanced oxidative stress, presents a serious risk factor for neurocognitive deficits, an illustration of accelerated ageing. Lead, administered to C57/B16 mice over 12 weeks, induced oxidative DNA damage and decreased cortical antioxidant biomarker as well as spatial learning and memory deficits with accompanying alterations to hippocampal BDNF-TrkB signaling ${ }^{27}$ these affected were alleviated by postexposure treatment with resveratrol. Finally, BDNF protects against the detrimental effects of acute stress. ${ }^{28,29}$ 
In the laboratory environment, several experimental models have been shown to accelerate the ageing process through disruptions in the normal functioning and integrity of BDNF. Social isolation/ deprivation of rats and mice induces chronic stress and may be applied usefully as a laboratory animal model for neuropsychiatric disorders, such as schizophrenia spectrum disorders and clinical depression. ${ }^{30,31}$ The decreased expression of BDNF has been observed generally as a consequence of these conditions with the downregulation of BDNF seemingly associated with increased anxiety-like symptoms/ behaviors among these rodents. ${ }^{32}$ In patients presenting newlydiagnosed multiple schlerosis, the observed cognitive dysfunction was most especially noticeable in male patients concomitant with reduced concentrations of BDNF in plasma. ${ }^{33}$ In the context of stress-related alcohol consumption history, it is evident that acute stress decreased serum BDNF with the quantitative family history of alcohol use disorder $(\mathrm{qFH})$ and age at first alcohol use in combination accounting for $15 \%$ of the variance in serum BDNF concentrations. ${ }^{34}$ Following a 12-week regime of Yoga and Meditation based lifestyle intervention to intervene against stress and cellular ageing, it was observed that levels of total antioxidant capacity, telomerase activity $\beta$-endorphin, BDNF and sirtuin-1 were increased markedly and reactive oxygen species, 8-hydroxy-2'-deoxyguanosine and interlekin-6, oxidative stress and pro-inflammatory biomarkers, after the Yoga and Meditation based lifestyle episode. ${ }^{35}$ Paired immunoglobulin-like receptor B is a functional receptor for myelin-associated inhibitory proteins that influences axon regeneration in injured brains. In an in vivo study of laboratory mice, it was observed that systemic (intraperitoneal) administration of TAT-PEP, a novel protein blocker of paired immunoglobulin-like receptor B which could pass through the blood brain barrier, had the effect of of enhancing motor capacity and spatial learning and memory in the animals, an effect that appeared to be mediated through regulation of BDNF secretion (Mi et al. ${ }^{36}$ Li et al. ${ }^{37}$ for TAT-PEP effects in induced-ischemia). Hypertension, with detrimental effects on the cerebral microcirculation thereby promoting an acceleration of cerebral aging, presents an independent risk factor for both vascular cognitive impairment and AD. In young C57BL/6 mice with induced hypertension through chronic (fourweeks) infusions of angiotensin II, It was found that hypertension and advanced ageing were related to the comparable decline in synaptic density in the stratum radiatum of the hippocampus of these mice, similar to ageing changes, the linked alterations of mRNA expression of several genes involved in regulation of neuronal function, including down-regulation of BDNF, Homer1, and Dlg4 was affected by hypertension influences..$^{38}$

Regional brain and serum BDNF levels and telomere length as common mediating factors and potential therapeutic targets for HIVassociated neurocognitive disorders and childhood trauma events offer illustrations of how the two biomarkers may be involved in the loss of health leading to premature ageing. ${ }^{39}$ Neurobiological trait abnormalities observed in individuals at genetic risk for bipolar depressive disorder may also presents biomarkers for accelerated ageing. For example, in a sample of bipolar patients and their siblings, telomere length was different between the three groups studied, patients, siblings and healthy volunteer controls with both patients and siblings showing a shorter telomeric length, $\mathrm{T} / \mathrm{S}$ ratio, compared with the healthy controls. ${ }^{40}$ Further, patients showed also increased levels of interleukin-6 and interleukin-10, pro-inflammatory cytokines, compared with the controls as well as increased levels of interleukin-6 and CCL24, myeloid progenitor inhibitory factor 2 encoded by the CCL24 gene, compared with their siblings. The C-C motif chemokine 11 levels were increased in siblings compared with the controls with similar tendencies found in the patients compared with the controls. Glutathione peroxidase activity was decreased also in the patients compared with the controls and siblings. Thus, even the unaffected siblings expressed features of accelerated ageing. Both DNA methylation, the addition of methyl groups to cytosines, and the shortening of telomeric length have emerged as indicators for an accelerated ageing and detrimental. In twenty-one medication-free male patients presenting mania and twenty age- and gender-matched healthy control volunteers, BDNF serum levels and telomeric were measured. ${ }^{41}$ The manic patients exhibited shorter telomere lengths than the control group; the telomere length of patients increased with lithium treatment. Patients in the late stages of disorder presented shorter telomere length than those patients in the early stages and the control group. hTERT gene expression, telomerase reverse transcriptase which comprises the most important unit in the telomerase complex, levels were up-regulated in the mania patients and remission compared with the control group, although BDNF level differences did not reach significance. In the laboratory, male rats that were exposed to prenatal stress exhibited higher levels of methylation (Bdnf IV) in the medial prefrontal cortex in comparison with the non-stressed male rat control group and stressed female rats. ${ }^{42}$ Furthermore, the prenatally-stressed rats presented shorter telomere lengths than the control group in the medial prefrontal cortex. These results provide evidence of the longterm impact of prenatal stress on brain DNA methylation and telomere biology thereby accelerating the ageing process with a deterioration of behavioral and health outcomes.

In conclusion, there is a burgeoning consensus that the levels of estimated BDNF lessen during disorder conditions and ill-health whereas suitably effective interventional therapies restore, and may even exceed the baseline levels of the neurotrophic factor. Representatively, voluntary aerobic exercise promoted non-spatial memory to a greater extent in males, forced exercise increased hippocampus-dependent learning and memory, and induced heightened levels of BDNF compared controls in male and female participants. ${ }^{43}$ Finally, physical exercise exerts an essential influence for the prevention and intervention against age-related cognitive decline and mild cognitive impairment, together with a multitude of neurologic and psychiatric conditions, including type II diabetes, hypertension, heart disease, stroke, osteoporosis, cancers, and obesity with exercise-boosted serum/regional BDNF a major avenue for disorder amelioration.

\section{Acknowledgements}

This Editorial is dedicated to the memory of Peter EK, Chairman of the Board Milmed AB, who was devoted to the study of healthy ageing and was instrumental in supporting the investigation of interventions to ameliorate the effects of neurodegenerative anabasis.

\section{Conflict of interest}

There is no conflict of interest in composing this manuscript.

\section{References}

1. Griva M, Lagoudaki R, Touloumi O, et al. Long-term effects of enriched environment following neonatal hypoxia-ischemia on behavior, BDNF and synaptophysin levels in rat hippocampus: Effect of combined treatment with G-CSF. Brain Res. 2017;1667:55-67. 
2. Li L, Deng B, Li S, et al. TAT-PEP, a novel blocker of PirB, enhances the recovery of cognitive function in mice after transient global cerebral ischemia. Behav Brain Res. 2017;30(326):322-330.

3. Śmieszek A, Stręk Z, Kornicka K, et al. Antioxidant and Anti-Senescence Effect of Metformin on Mouse Olfactory Ensheathing Cells (mOECs) May Be Associated with Increased Brain-Derived Neurotrophic Factor Levels-An Ex Vivo Study. Int J Mol Sci. 2017;18(4).

4. Markham A, Bains R, Franklin P, et al. Changes in mitochondrial function are pivotal in neurodegenerative and psychiatric disorders: how important is BDNF?. Br J Pharmacol. 2014;171(8):2206-2229.

5. Siuda J, Patalong-Ogiewa M, Żmuda W, et al. Cognitive impairment and BDNF serum levels. Neurol Neurochir Pol. 2017;51(1):24-32.

6. Marston KJ, Newton MJ, Brown BM, et al. Intense resistance exercise increases peripheral brain-derived neurotrophic factor. J Sci Med Sport. 2017;1440-2440.

7. Venezia AC, Quinlan E, Roth SM. A single bout of exercise increases hippocampal Bdnf: Influence of chronic exercise and noradrenalin. Genes Brain Behav. 2017.

8. Sullivan AM, O'Keeffe GW. Neurotrophic factor therapy for Parkinson's disease: past, present and future. Neural Regen Res. 2016;11(2):205-207.

9. Bothwell M. Recent advances in understanding neurotrophin signaling F1000Res. 2016;28(5);1885.

10. Ibáñez CF, Andressoo JO. Biology of GDNF and its receptors - Relevance for disorders of the central nervous system. Neurobiol Dis. 2017;97(Pt B):80-89.

11. Sampaio TB, Savall AS, Gutierrez MEZ, et al. Neurotrophic factors in Alzheimer's and Parkinson's diseases: implications for pathogenesis and therapy. Neural Regen Res. 2017;12(4):549-557.

12. Boots EA, Schultz SA, Clark LR, et al. BDNF Val66Met predicts cognitive decline in the Wisconsin Registry for Alzheimer's Prevention. Neurology. 2017;88(22):2098-2106.

13. Xia DY, Huang $\mathrm{X}, \mathrm{Bi} \mathrm{CF}$, et al. PGC- $1 \alpha$ or FNDC5 Is Involved in Modulating the Effects of A $\beta 1-42$ Oligomers on Suppressing the Expression of BDNF, a Beneficial Factor for Inhibiting Neuronal Apoptosis, A $\beta$ Deposition and Cognitive Decline of APP/PS1 Tg Mice. Front Aging Neurosci. 2017;21(9):65.

14. Holmes SE, Esterlis I, Mazure CM, et al. $\beta$-Amyloid, APOE and BDNF Genotype, and Depressive and Anxiety Symptoms in Cognitively Normal Older Women and Men. Am J Geriatr Psychiatry. 2016;24(12):1191-1195.

15. Koppel SJ, Swerdlow RH. Neuroketotherapeutics: A modern review of a century-old therapy. Neurochem Int. 2017;S0197-0186(17):30227-30 229.

16. Durand D, Carniglia L, Turati J, et al. Amyloid-beta neurotoxicity and clearance are both regulated by glial group II metabotropic glutamate receptors. Neuropharmacology. 2017;1(123):274-286.

17. Besga A, Chyzhyk D, Echeveste J, et al. White Matter Tract Integrity in Alzheimer's Disease vs. Late Onset Bipolar Disorder and Its Correlation with Systemic Inflammation and Oxidative Stress Biomarkers. Front Aging Neurosci. 2017;16(9):179.

18. Rea IM. Towards ageing well: Use it or lose it: Exercise, epigenetics and cognition. Biogerontology. 2017;18(4):679-691.

19. Nigam SM, Xu S, Kritikou JS, et al. Exercise and BDNF reduce A $\beta$ production by enhancing $\alpha$-secretase processing of APP. J Neurochem. 2017;142(2):286-296.
20. Wang CN, Wang YJ, Wang $\mathrm{H}$, et al. The Anti-dementia Effects of Donepezil Involve miR-206-3p in the Hippocampus and Cortex. Biol Pharm Bull. 2017;40(4):465-472.

21. Zaletel I, Filipović D, Puškaš N. Hippocampal BDNF in physiological conditions and social isolation. Rev Neurosci. 2017.

22. Zheng J, Jiang YY, Xu LC, et al. Adult Hippocampal Neurogenesis along the Dorsoventral Axis Contributes Differentially to Environmental Enrichment Combined with Voluntary Exercise in Alleviating Chronic Inflammatory Pain in Mice. J Neurosci. 2017;37(15):4145-4157.

23. Borba EM, Duarte JA, Bristot G, et al. Brain-Derived Neurotrophic Factor Serum Levels and Hippocampal Volume in Mild Cognitive Impairment and Dementia due to Alzheimer Disease. Dement Geriatr Cogn Dis Extra. 2016;6(3):559-567.

24. Badowska-Szalewska E, Ludkiewicz B, Krawczyk R, et al. Comparison of the influence of two models of mild stress on hippocampal brainderived neurotrophin factor (BDNF) immunoreactivity in old age rats. Acta Neurobiol Exp (Wars). 2017;77(1):68-76.

25. Ghanbari A, Ghareghani M, Zibara K, et al. Light-Emitting Diode (LED) therapy improves occipital cortex damage by decreasing apoptosis and increasing BDNF-expressing cells in methanol-induced toxicity in rats. Biomed Pharmacother. 2017;89:1320-1330.

26. Balietti M, Giuli C, Fattoretti P, et al. Effect of a Comprehensive Intervention on Plasma BDNF in Patients with Alzheimer's Disease. $J$ Alzheimers Dis. 2017;57(1):37-43

27. Zhang L, Tu R, Wang Y, et al. Early-Life Exposure to Lead Induces Cognitive Impairment in Elder Mice Targeting SIRT1 Phosphorylation and Oxidative Alterations. Front Physiol. 2017;29(8):446.

28. Passaro A, Soavi C, Marusic U, et al. Computerized cognitive training and brain derived neurotrophic factor during bed rest: mechanisms to protect individual during acute stress. Aging (Albany NY). 2014;9(2):393-407.

29. Raefsky SM, Mattson MP. Adaptive responses of neuronal mitochondria to bioenergetic challenges: Roles in neuroplasticity and disease resistance. Free Radic Biol Med. 2017;102:203-216.

30. Arcego DM, Toniazzo AP, Krolow R, et al. Impact of High-Fat Diet and Early Stress on Depressive-Like Behavior and Hippocampal Plasticity in Adult Male Rats. Mol Neurobiol. 2017.

31. Lampert C, Arcego DM, Pereira CN, et al. Short post-weaning social isolation induces long-term changes in the dopaminergic system and increases susceptibility to psychostimulants in female rats. Int $J$ Dev Neurosci. 2017;61:21-30.

32. Murínová J, Hlaváčová N, Chmelová M, et al. The Evidence for Altered BDNF Expression in the Brain of Rats Reared or Housed in Social Isolation: A Systematic Review. Front Behav Neurosci. 2017;31(11):101.

33. Prokopova B, Hlavacova N, Vlcek M, et al. Early cognitive impairment along with decreased stress-induced BDNF in male and female patients with newly diagnosed multiple sclerosis. J Neuroimmunol. 2017;302:34-40

34. Sharma S, Graham R, Rohde R, et al. Stress-induced change in serum BDNF is related to quantitative family history of alcohol use disorder and age at first alcohol use. Pharmacol Biochem Behav. 2017;153:12-17.

35. Tolahunase M, Sagar R, Dada R. Impact of Yoga and Meditation on Cellular Aging in Apparently Healthy Individuals: A Prospective, OpenLabel Single-Arm Exploratory Study. Oxid Med Cell Longev. 2017:1-9.

36. Mi YJ, Chen H, Guo N, et al. Inhibition of PirB Activity by TAT-PEP Improves Mouse Motor Ability and Cognitive Behavior. Front Aging Neurosci. 2017;20(9):199. 
37. Li JY, Liu J, Manaph NPA, et al. ProBDNF inhibits proliferation, migration and differentiation of mouse neural stem cells. Brain Res 2017;1(1668):46-55.

38. Tucsek Z, Tarantini S, Yabluchanskiy A, et al. Hypertension-induced synapse loss and impairment in synaptic plasticity in the mouse hippocampus mimics the aging phenotype: implications for the pathogenesis of vascular cognitive impairment. Geroscience. 2017.

39. Womersley JS, Seedat S, Hemmings SMJ. Childhood maltreatment and HIV-associated neurocognitive disorders share similar pathophysiology: a potential sensitisation mechanism? Metab Brain Dis. 2017.

40. Vasconcelos MMP, Fries GR, Gubert C, et al. Telomere Length, Oxidative Stress, Inflammation and BDNF Levels in Siblings of Patients with Bipolar Disorder: Implications for Accelerated Cellular Aging. Int $J$ Neuropsychopharmacol. 2017;20(6):445-454.
41. Cinar KR. Telomere length and hTERT in mania and subsequent remission. Rev Bras Psiquiatr. 2017.

42. Blaze J, Asok A, Borrelli K, et al. Intrauterine exposure to maternal stress alters Bdnf IV DNA methylation and telomere length in the brain of adult rat offspring. Int J Dev Neurosci. 2017;S0736-5748(16):30374-30374.

43. Barha CK, Davis JC, Falck RS, et al. Sex differences in exercise efficacy to improve cognition: A systematic review and meta-analysis of randomized controlled trials in older humans. Front Neuroendocrinol. 2017;46:71-85. 